\title{
ELECTRICAL CAPACITANCE MEASUREMENT BY SCATTER ELLIPSE APPROXIMATION
}

\author{
Ivan Kostiukov, PhD, Senior Lecturer \\ National Technical University "Kharkiv Polytechnic Institute”, Ukraine, \\ email: iakostiukow@gmail.com
}

https://doi.org/10.23939/istcmtm2020.03.

\begin{abstract}
This article is devoted to the substantiation of the possibility of electrical capacitance measurement utilizing equations that are based on the approximation of scattering ellipse, formed by signals proportional to the current flowing through the capacitive tested object and voltage drop on the tested object. On the contrary, to previously developed algorithms, which were based on the approximation of the scattering ellipse by applying the least-squares method, in this case, the approximation was carried out by simply using the values of signals amplitude directly determined from current and voltage curves. The value of the phase shift between the current and voltage curves, which is also necessary to approximate the shape of the scattering ellipse, was determined by using the cross-correlation method. Besides, the article provides formulas for calculating the reactive component of voltage drop on the tested object which is based on the approximation of scattering ellipse without using the least-squares method. Formulas for calculation of the reactive component of the voltage drop on the tested object after the reduction of the quadratic form of the elliptic curve to its canonical form are also given. The results of the impact of the reduction of the quadratic form of scattering ellipse to its canonical form on the value of correlation coefficient between sine curves of current and voltage as well as on the magnitude of major semi-axis and minor semi-axis of scattering ellipse are illustrated. Also, it was shown the relationship between the values of the reactive component of voltage drop on the capacitive tested object, which were determined before the reducing of the quadratic form of scattering ellipse to its canonical form and after such reducing. Despite the rejection of the applying of the least-squares method, to simplify the calculation algorithms, and also despite the presence of a significant noise component in sampled and processed curves of current and voltage, the experimental test has shown the sufficient level of accuracy and, consequently, the possibility of measuring the electric capacitance by approximating the scatter ellipse employing parameters of the quadratic form directly calculated from previously sampled sine curves of current and voltage.
\end{abstract}

Key words: Phase shift, Correlation coefficient, Quadratic form, Accuracy of measurement, Quality factor.

\section{Introduction}

The problem of accurate measurement of impedance components is actual in various applied problems in engineering and medicine. Typical examples of such problems are eddy-current testing [1], diagnostics of the functioning of the human body [2,3], measurement of magnetic properties of ferromagnetic materials [4], the analysis of signals obtained from capacitive transducers [5]. Due to the sensitivity of electrical capacitance to the extent of moistening special attention to the problem of impedance measurement is paid in issues that regard the assessment of the technical state of electrical insulation $[6,7]$. Due to the close linkage with polarization processes, the value of electrical capacitance affects the relaxation currents in electrical insulation [8]. In the majority of cases of electrical insulation testing, which require carrying out of measurements of electrical insulation parameters under the pretty low values of tested voltage, such measurements can be made by applying conventional equipment and conventional techniques that are used in the general practice of carrying out impedance or immittance measurements. Typical approaches for impedance measurement imply the applying of various schemes of $\mathrm{AC}$ bridges or schemes with the current comparator [9]. A significant amount of measurement devices are based on the applying of the ammeter-voltmeter method. In this case, the value of impedance is calculated as a ratio of voltage on the tested object to the value of the current, which flows through the tested object. Impedance components are determined by synchronous detection. The example of practical implementation of this technique is the E7-25 immittance meter, which was used in [4] for the measurements of complex magnetic permeability. Measurement of electrical insulation parameters can be also accomplished utilizing various measurement schemes based on the applying of phase detectors with phase-shifting circuits $[5,10]$, or, according to the terminology of [11], measurement schemes with quasibalanced circuits. Some other approaches to impedance measurement imply the applying of Fourier transform. Some disadvantages of such an approach are caused by comparably sophisticated implementation due to the high requiems to the size of memory which is necessary for hardware realization and a substantial number of mathematical operations. Mentioned problems can be settled by applying the particular sampling method, described in [12]. Impedance measurements can also be made based on applying the AD5933 integrated circuit, which contains a direct digital synthesis sine signal generator, digital-to-analog convertor, programmable gain amplifier, and unit for Fourier transform implementation. This approach for impedance measurements was applied in [13]. Some other recent studies have been focused on the adaptation of scattering ellipse fitting algorithms and their practical implementation [14-17]. These algorithms are based on the estimation of phase shift and amplitudes of voltage drop on the reference 
impedance and the unknown impedance. The values of amplitudes and phase shift between the analyzed signals are obtained by solving the least-squares problem by applying modified in [18] algorithm.

\section{Shortcomings}

Despite the high accuracy of the ellipse fitting method, the implementation of a numerically stable algorithm, which is necessary for ellipse fitting, complicates the software development and causes additional requirements to the technical properties of digital signal processing hardware device.

\section{Goal of the Study}

The applying of the ellipse fitting method, based on the direct calculation of parameters of a quadratic form for scattering ellipse, without any preliminary processing of sampled sine curves of current and voltage by the least-squares method, for electrical capacitance measurement.

\section{Studies of the Methods of Impedance Measurement}

The method of impedance measurement by scattering ellipse fitting in its original form is based on the analysis of expressions for the values of voltage drop on reference impedance $u_{1}(t)$ and measured impedance $u_{2}(t)[14]$ :

$$
\begin{aligned}
& u_{1}(t)=D_{1} \cos \left(2 \pi f t+\varphi_{1}\right)+C_{1} ; \\
& u_{2}(t)=D_{2} \cos \left(2 \pi f t+\varphi_{2}\right)+C_{2},
\end{aligned}
$$

where $C_{1}, C_{2}$ denote the $D C$ components of signals, $f$ is the frequency of applied voltage, $D_{1}, D_{2}, \varphi_{1}, \varphi_{2}$ respectively denote amplitudes and initial phases of signals.

In this case, the quadratic form for the elliptic curve which is determined as $u_{2}=f\left(u_{1}\right)$ can be written in the following form [14]:

$$
\begin{gathered}
\left(\frac{u_{1}-C_{1}}{D_{1}}\right)^{2}+\left(\frac{u_{2}-C_{2}}{D_{2}}\right)^{2}- \\
-2 \frac{\left(u_{1}-C_{1}\right)\left(u_{2}-C_{2}\right)}{D_{1} D_{2}} \cos (\Delta \varphi)-\sin ^{2}(\Delta \varphi)=0 .
\end{gathered}
$$

where $\Delta \varphi$ denotes the value of the phase shift between sine signals. It should be noted that the form of (3) is similar to the form of the expression for scattering ellipse in cross-section of probability density distribution function of two random variables, which is obtained by the intersection of two-dimensional normal distribution function by planes which are parallel to the $x O y$ plane [19]:

$$
\frac{\left(x-m_{x}\right)^{2}}{\sigma_{x}^{2}}+\frac{\left(y-m_{y}\right)^{2}}{\sigma_{y}^{2}}-\frac{2 r\left(x-m_{x}\right)\left(y-m_{y}\right)}{\sigma_{x} \sigma_{y}}=\lambda^{2},
$$

where $r$ is the value of correlation coefficient between two random values $x$ and $y, m_{x}$ and $m_{y}$ respectively represent the values of mathematical expectation of random variables $x$ and $y, \sigma_{x}$ and $\sigma_{y}$ denote standard deviations of $x$ and $y$. After the comparison of (3) and (4), it can be easily noticed that the form of (3) is pretty similar to the form of (4). Such similarity is caused by the analogy between the values of the correlation coefficient, determined for two sine functions with some value of phase shift, and the value of their power factor:

$$
r=\cos (\Delta \varphi)=\frac{R_{u 1 u 2}(0)}{R_{u 1 u 1}(0) R_{u 2 u 2}(0)},
$$

where $R_{u 1 u 2}(0)$ is the value of cross-correlation function for $u_{1}(t)$ and $u_{2}(t)$, determined for zero value of its argument, $R_{u 1 u 1}(0)$ and $R_{u 2 u 2}(0)$ are the values of autocorrelation functions of $u_{1}(t)$ and $u_{2}(t)$ determined for zero value of lag time. As the values of correlation coefficient $r$ and $\cos (\Delta \varphi)$ can be easily obtained from (5) the value of $\lambda$ in (4) can be obtained by using (6):

$$
\lambda=\sin (\Delta \varphi)=\sqrt{1-r^{2}} .
$$

The value of electrical capacitance can be determined after the calculation of the reactive component of applied to the tested object voltage $u_{2 r}$. In this paper, two different approaches to the calculation of $u_{2 r}$ are considered. The first is based on the reduction of (3) to its canonical form. The second is based on the direct calculation of $u_{2 r}$, by solving (3) concerning $u_{2}$. The conventional approach for the reduction of (3) to its canonical form implies the determination of eigenvalues and eigenvectors for the matrix build for quadratic form (3) with a further selection of a new orthogonal basis in which (3) will have a simplified form. Such a simplified form usually allows us to get rid of the term which contains multiplication of $u_{1}$ and $u_{2}$. The orthogonal matrix of variables replacement consists of normalized eigenvectors of the matrix for the quadratic form (3) in case if its eigenvalues do not coincide with each other. In expanded form (3) can be written according to (7):

$$
\begin{gathered}
\frac{u_{1}^{2}}{D_{1}^{2}}-2 \frac{u_{1} C_{1}}{D_{1}^{2}}+\frac{C_{1}^{2}}{D_{1}^{2}}+\frac{u_{2}^{2}}{D_{2}^{2}}-2 \frac{u_{2} C_{2}}{D_{2}^{2}}+\frac{C_{2}^{2}}{D_{2}^{2}}-\frac{2 r u_{1} u_{2}}{D_{1} D_{2}}+. \\
+\frac{2 r u_{1} C_{2}}{D_{1} D_{2}}+\frac{2 r C_{1} u_{2}}{D_{1} D_{2}}-\frac{2 r C_{1} C_{2}}{D_{1} D_{2}}-\lambda^{2}=0 .
\end{gathered}
$$

By appropriate combining terms with $u_{1}$ and $u_{2}$, (7) can be reduced to the following expression:

$$
a_{11} u_{1}^{2}+2 a_{12} u_{1} u_{2}+a_{22} u_{2}+B u_{1}+C u_{2}+D=0,
$$

where all coefficients can be determined according to (9-14):

$$
\begin{aligned}
& a_{11}=\frac{1}{D_{1}^{2}} ; \\
& a_{22}=\frac{1}{D_{2}^{2}} ;
\end{aligned}
$$




$$
\begin{gathered}
a_{12}=-\frac{r}{D_{1} D_{2}} ; \\
B=-\frac{2 C_{1}}{D_{1}^{2}}+2 \frac{r C_{2}}{D_{1} D_{2}} ; \\
C=-\frac{2 C_{2}}{D_{2}^{2}}+2 \frac{r C_{1}}{D_{1} D_{2}} ; \\
D=\frac{C_{1}^{2}}{D_{1}^{2}}+\frac{C_{2}^{2}}{D_{2}^{2}}-\frac{2 r C_{1} C_{2}}{D_{1} D_{2}}-\lambda^{2} .
\end{gathered}
$$

Taking into consideration comparably small order of square matrix for quadratic form (8), which contains only terms $\alpha_{11}, \alpha_{12}$, and $\alpha_{22}$, its eigenvalues can be determined by using an analytical solution. For this purpose it is suitable to apply the following expression [20]:

$$
\begin{gathered}
\lambda_{1,2}=0.5\left[\left(\alpha_{11}+\alpha_{22}\right) \pm\right. \\
\left. \pm \sqrt{\left(\alpha_{11}+\alpha_{22}\right)^{2}-4\left(\alpha_{11} \alpha_{22}-\alpha_{12}^{2}\right)}\right] .
\end{gathered}
$$

The transformation of coordinate axes $u_{1}$ and $u_{2}$ changes the values of coefficients $D, B, C$ in (8). The impact of variables substitution on the value of $D$ in (8) can be determined according to (16) [21]:

$$
D_{1}=D-\frac{B_{1}^{2}}{4 \lambda_{1}}-\frac{C_{1}^{2}}{4 \lambda_{2}},
$$

where $D_{1}$ designates the value of $D$ after the variables substitution. The values of coefficients $B$ and $C$ after the variables substitution can be determined according to (17-18):

$$
\begin{gathered}
B_{1}=B \beta_{11}+C \beta_{12} ; \\
C_{1}=B \beta_{21}+C \beta_{22},
\end{gathered}
$$

where $\beta_{11}, \beta_{12}, \beta_{21}, \beta_{22}$ denote eigenvectors that correspond to eigenvalues determined according to (15).

Calculation of $B_{1}, C_{1}$, and $D_{1}$ enables the determination of the length of semi-major and semiminor axes of the elliptic curve. This calculation can be accomplished by using (19-20) [21]:

$$
\begin{gathered}
a=\sqrt{-\frac{D_{1}}{\lambda_{1}}} ; \\
b=\sqrt{-\frac{D_{1}}{\lambda_{2}}},
\end{gathered}
$$

where $a$ and $b$ denote the length of semi-major and semiminor axes of the elliptic curve obtained after axes transformation. The solution of (7) concerning $u_{2}$ enables to obtain the expression which can be used to determine the accuracy of ellipse fitting by using the value of phase shift determined according to (5):

$$
\begin{gathered}
u_{2}=\frac{D_{1} C_{2}-r D_{2} C_{1}+r D_{2} u_{1}}{D_{1}} \pm \\
\pm \frac{\sqrt{p_{1}+p_{2}+p_{3}+p_{4}+p_{5}+p_{6}+p_{7}}}{D_{1}}
\end{gathered}
$$

where all coefficients $p_{1} . . p_{7}$ can be determined by applying (22-28):

$$
\begin{gathered}
p_{1}=r^{2} D_{2}^{2} C_{1}^{2} ; \\
p_{2}=-2 r^{2} D_{2}^{2} C_{1} u_{1} ; \\
p_{3}=r^{2} D_{2}^{2} u_{1}^{2} ; \\
p_{4}=-D_{2}^{2} u_{1}^{2} ; \\
p_{5}=2 D_{2}^{2} u_{1} C_{1} ; \\
p_{6}=-D_{2}^{2} C_{1}^{2} ; \\
p_{7}=\lambda^{2} D_{1}^{2} D_{2}^{2} ;
\end{gathered}
$$

The variables substitution made to reduce the quadratic form (8) to its canonical form allows us to carry out the appropriate rotation of axes $u_{1}$ and $u_{2}$ after which analyzed signals become orthogonal. This circumstance simplifies the determination of the reactive component of applied voltage which is caused by the voltage drop on measured capacitance, as in this case, the value of the reactive component of the applied voltage is equal to the value of one of the semi-axes of the elliptic curve. However, the value of the reactive component of applied voltage in the transformed coordinate system does not necessarily coincide with the value of the reactive component in the original coordinate system. By substituting $u_{1}=0$ in (21) is possible to derive the expression, which can be used for the calculation of reactive component applied to the tested sample voltage:

$u_{2 r}=-\frac{-D_{1} C_{2}+r D_{2} C_{1}-\sqrt{D_{2}^{2}\left(r^{2} C_{1}^{2}-C_{1}^{2}+\lambda^{2} D_{1}^{2}\right)}}{D_{1}}$.

By replacing the value of $\lambda$ in (7) with its value from (6), the expression for the reactive component of voltage can be also written in the alternative form:

$$
u_{2 r}=-\frac{-D_{1} C_{2}+r D_{2} C_{1}-\sqrt{D_{2}^{2}\left(r^{2}-1\right)\left(C_{1}^{2}-D_{1}^{2}\right)}}{D_{1}} .
$$

By taking into consideration (30), in case of absence or negligible mean values $C_{1}$ and $C_{2}$, the expression for $u_{2 r}$ can be reduced to (31):

$$
u_{2 r}=\frac{\sqrt{D_{1}^{2} D_{2}^{2}-D_{1}^{2} D_{2}^{2} r^{2}}}{D_{1}} .
$$

Before the variables substitution, the value of phase shift between the current and voltage curves can be determined according to (5), therefore the value of correlation coefficient $r$ is determined by a ratio of the active component of an applied voltage to the reactive component of an applied voltage. After the variables substitution, sine curves of current and voltage become orthogonal and, therefore, the value of correlation coefficient $r$ in (31) is equal to zero. In this case, the main axes of the elliptic curve coincide with the axes of the coordinate system. In the original coordinate system, 
for correlated curves of current and voltage, the values of their amplitudes do not coincide with the values of semiaxes of the elliptic curve. In the transformed coordinate system semi-axes of the elliptic curve coincide with the axes of the coordinate system. Therefore, the values of $a$ and $b$, which are determined according to (19) and (20), coincide with the values of amplitudes of transformed signals $u_{1}$ and $u_{2}$. Taking into consideration the zero value of the correlation coefficient and coincidence of $a$ and $b$ with the amplitudes of transformed signals $u_{1}$ and $u_{2}$, (31) can be reduced to the following expression:

$$
u_{2 r}=\frac{a b}{D_{1}} \text {. }
$$

Therefore, the value of the reactive component of applied voltage in the general case can be determined according to (30). In the case of negligible mean values of $u_{1}$ and $u_{2}$, before the coordinate system transformation, the value of the reactive component can be determined according to (31). After the coordinate system transformation by reducing quadratic form (8) to its canonical form, the value of the reactive component can be determined according to (32). After the determination of the reactive component of applied voltage $u_{2 r}$, the value of electrical capacitance $C_{\mathrm{s}}$ for the series equivalent scheme of the tested object can be determined as:

$$
C_{s}=\frac{D_{1}}{\omega u_{2 r}} \text {. }
$$

\section{Results and Implementation of the}

\section{Method}

5.1 Illustration of the impact of coordinate system transformation on the form of elliptic curve

The results of mathematical modeling have been obtained by generating sine curves $u_{1}$ and $u_{2}$ in MATLAB.

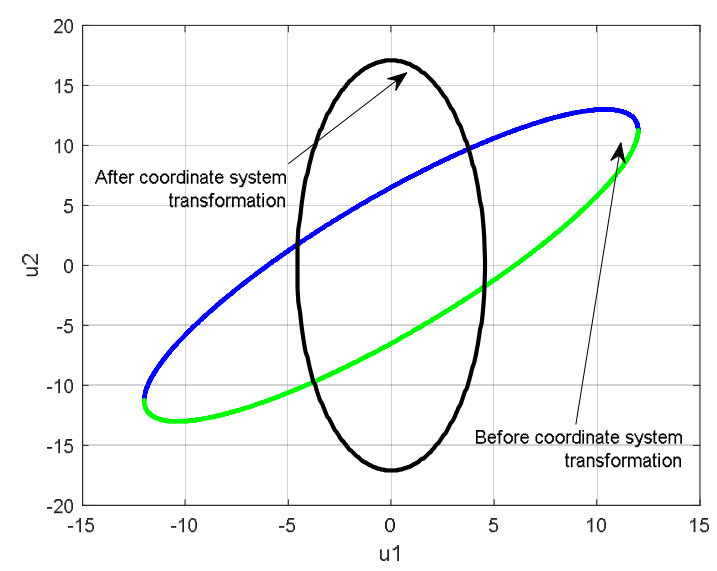

Fig. 1. Impact of coordinate system transformation caused by the reducing of quadratic form to its canonical form for the value of phase shift between $u_{1}$ and $u_{2}$ equal to $\pi / 6, a=17.093$, $b=4.563$
Two generated curves were supposed to represent voltage signals, one of which is obtained from a converter that converts current which flows through the tested object to voltage, the other represents the voltage on the outputs of the tested object. Figures $1-2$ illustrate the distinction between the form of elliptic curves and values of amplitudes of generated sine curves before and after the axis transformation.

While before the coordinate system transformation the amplitudes were equal to the values of $12 \mathrm{~V}$ and $13 \mathrm{~V}$ after the axes transformation corresponding values have been changed to the $17.093 \mathrm{~V}$ and $4.563 \mathrm{~V}$ for the value of phase shift equal to $\pi / 6$, and the $13.806 \mathrm{~V}$ and 11.063 $\mathrm{V}$ for the value of phase shift equal to $\pi / 2.3$.

\subsection{Comparative analysis of expressions for} the calculation of reactive component of voltage

As the value of the reactive component of applied to the tested object voltage can be determined by using different expressions, a comparative analysis of their accuracy was also carried out. The values of amplitudes were set equal to: $D_{1}=3$ Volt, $\mathrm{D}_{2}=13$ Volt. The results of the comparative calculations are given in Table 1.

As can be concluded from the results of the calculation in Table 1, the increase in the number of points of analyzed signals causes the increasing accuracy of calculations. For both cases, the decreasing phase shift leads to the increasing of relative error of calculation. Consequently, for tested objects with a high-quality factor, the accuracy of electrical capacitance measurement is supposed to be higher in comparison with tested objects with a low-quality factor. The mentioned circumstance causes the dependence of the accuracy of calculations on the technical state of the tested capacitive object.

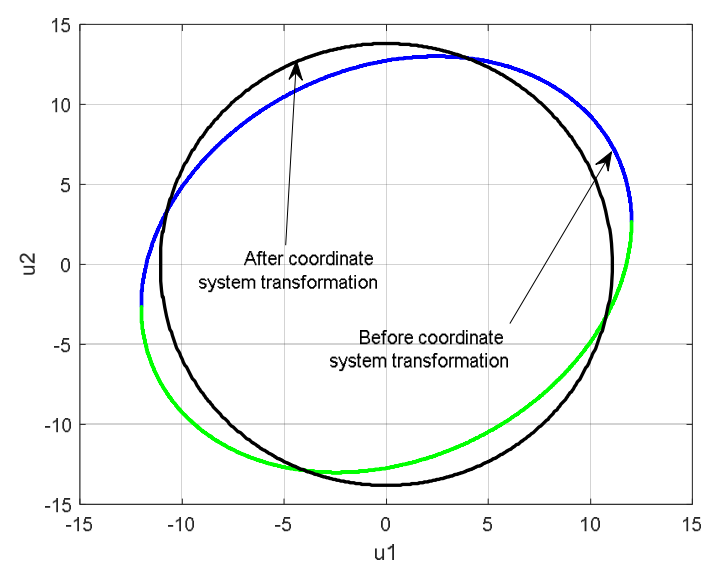

Fig. 2. Impact of coordinate system transformation caused by the reducing of quadratic form to its canonical form for the value of phase shift between $u_{1}$ and $u_{2}$ equal to $\pi / 2.3, a=$ $13.806, b=11.063$ 
The results of the comparative analysis of different expressions for the calculation of reactive component of the applied voltage

\begin{tabular}{|c|c|c|c|c|c|}
\hline \multicolumn{6}{|c|}{ For the number of points of analyzed signals equal to 210} \\
\hline Phase shift & $\begin{array}{c}\text { Reactive } \\
\text { component, Volt }\end{array}$ & $\begin{array}{c}\text { Calculation } \\
\text { according to (30), } \\
\text { Volt }\end{array}$ & $\begin{array}{c}\text { Calculation } \\
\text { according to (32), } \\
\text { Volt }\end{array}$ & $\begin{array}{l}\text { The relative error } \\
\text { of calculation } \\
\text { according to (32) }\end{array}$ & $\begin{array}{l}\text { The relative error } \\
\text { of calculation } \\
\text { according to (30) }\end{array}$ \\
\hline $99.78^{\circ}$ & 12.9999 & 13.1291 & 13.0644 & $0.4963 \%$ & $0.9938 \%$ \\
\hline $85.71^{\circ}$ & 12.9636 & 13.0925 & 13.0280 & $0.4963 \%$ & $0.9938 \%$ \\
\hline $78.26^{\circ}$ & 12.7281 & 12.8546 & 12.7913 & $0.4964 \%$ & $0.9939 \%$ \\
\hline $62.07^{\circ}$ & 11.4857 & 11.5999 & 11.5427 & $0.4968 \%$ & $0.9943 \%$ \\
\hline $56.25^{\circ}$ & 10.8091 & 10.9166 & 10.8628 & $0.4970 \%$ & $0.9946 \%$ \\
\hline \multicolumn{6}{|c|}{ For the number of points of analyzed signals equal to 21} \\
\hline Phase shift & $\begin{array}{c}\text { Reactive } \\
\text { component }\end{array}$ & $\begin{array}{c}\text { Calculation } \\
\text { according to (30), } \\
\text { Volt }\end{array}$ & $\begin{array}{c}\text { Calculation } \\
\text { according to (32), } \\
\text { Volt }\end{array}$ & $\begin{array}{l}\text { The relative error } \\
\text { of calculation } \\
\text { according to ( } 32)\end{array}$ & $\begin{array}{l}\text { The relative error } \\
\text { of calculation } \\
\text { according to }(30)\end{array}$ \\
\hline $99.78^{\circ}$ & 12.9999 & 14.2239 & 13.6049 & $4.6536 \%$ & $9.4155 \%$ \\
\hline $85.71^{\circ}$ & 12.9636 & 14.1844 & 13.5671 & $4.6548 \%$ & $9.4167 \%$ \\
\hline $78.26^{\circ}$ & 12.7281 & 13.9276 & 13.3215 & $4.6622 \%$ & $9.4241 \%$ \\
\hline $62.07^{\circ}$ & 11.4857 & 12.5724 & 12.0255 & $4.6999 \%$ & $9.4618 \%$ \\
\hline $56.25^{\circ}$ & 10.8091 & 11.8339 & 11.3192 & $4.7193 \%$ & $9.4812 \%$ \\
\hline \multicolumn{6}{|c|}{ For the number of points of analyzed signals equal to 2100} \\
\hline Phase shift & $\begin{array}{c}\text { Reactive } \\
\text { component }\end{array}$ & $\begin{array}{c}\text { Calculation } \\
\text { according to (30), } \\
\text { Volt }\end{array}$ & $\begin{array}{c}\text { Calculation } \\
\text { according to (32), } \\
\text { Volt }\end{array}$ & $\begin{array}{l}\text { The relative error } \\
\text { of calculation } \\
\text { according to (32) }\end{array}$ & $\begin{array}{l}\text { The relative error } \\
\text { of calculation } \\
\text { according to }(30)\end{array}$ \\
\hline $99.78^{\circ}$ & 12.9999 & 13.0129 & 13.0064 & $0.0500 \%$ & $0.0999 \%$ \\
\hline $85.71^{\circ}$ & 12.9636 & 12.9766 & 12.9701 & $0.0500 \%$ & $0.0999 \%$ \\
\hline $78.26^{\circ}$ & 12.7281 & 12.7408 & 12.7345 & $0.0500 \%$ & $0.0999 \%$ \\
\hline $62.07^{\circ}$ & 11.4857 & 11.4971 & 11.4914 & $0.0500 \%$ & $0.0999 \%$ \\
\hline $56.25^{\circ}$ & 10.8091 & 10.8199 & 10.8145 & $0.0500 \%$ & $0.0999 \%$ \\
\hline
\end{tabular}

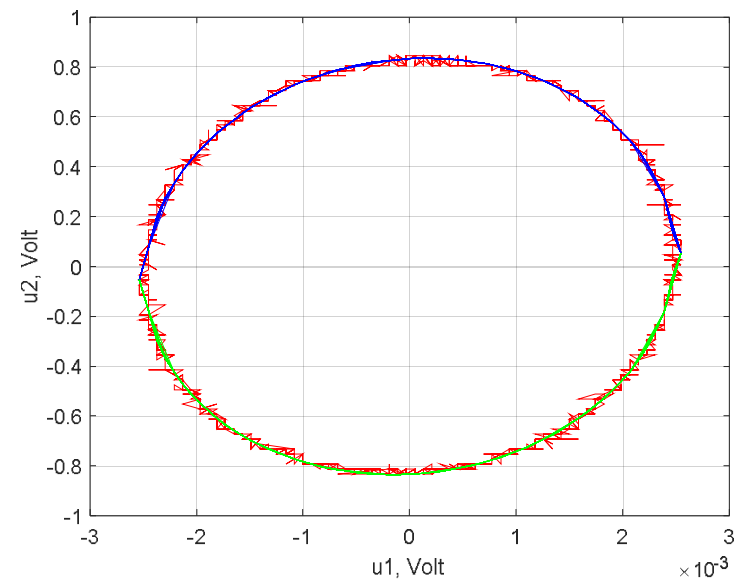

Fig. 3. The results of the elliptic curve approximation by direct calculation of quadratic form parameters

\subsection{Results of implementation}

Practical implementation of electrical capacitance measurement by scattering ellipse fitting was carried out by comparison of the results of electrical capacitance measurement by applying the described processing of experimental data and RLC meter DE-5000. The value of the frequency of applied voltage was set equal to 100
Hz. For this frequency, the measured value of the quality factor of the tested capacitor was equal to 32 .

The results of electrical capacitance calculation were: $4.71 \cdot 10^{-6} \mathrm{~F}$, after the applying of (32) for the reactive component of voltage calculation and $4.74 \cdot 10^{-6}$ F, after the applying of (30) for reactive component calculation, whereas the result of direct measurement was equal to $4.69 \cdot 10^{-6} \mathrm{~F}$.

\section{Conclusions}

The proposed expressions for electrical capacitance calculation are based on fitting the scattering ellipse build by processing curves of current which flows through the tested object and voltage drop on the outputs of the tested object. The direct calculation of parameters of quadratic form for the scattering ellipse, without the applying of the least-squares method, enables the simplification of the applying of the ellipse fitting algorithm for electrical capacitance measurement. In this case, the accuracy of fitting depends on the value of the phase shift between signals. Despite the presence of noisy components in analyzed signals, the refusal of the applying of the least-squares method did not lead to significant loss of accuracy. This might be caused by the 
fact that the highest level of noise immunity of the crosscorrelation method for phase shift measurement, which was used for calculation of this parameter in quadratic form, usually can be observed for signals with the value of phase shift close to $90^{\circ}$. Therefore, for tested objects with high-quality factors mentioned peculiarity of the cross-correlation method for phase shift measurement can cause the increase of accuracy in case of direct calculation of quadratic form parameters and refusal of the applying of the least-squares method.

According to the results of comparative analysis of obtained expressions for the reactive component of applied voltage, the reduction of quadratic form for the elliptic curve to its canonical form also allows decreasing of the source of the error caused by the presence of DC components in analyzed signals.

\section{Conflict of Interests}

The author does not declare financial or any other conflicts of interest concerning this article.

\section{References}

[1] J. Garcia-Martin, J. Gomez-Gil, E. VazquesSqnchez, "Non-destructive techniques based on eddy current testing", Sensors, 17: 2525-2565. doi:10.3390/s110302525

[2] O. Naumovich, Y. Pokhodylo, M. Dovhan, "Modeling of human body tissues impedance components in frequency range", Measuring Equipment and Metrology, no. 80, p. 49-53, 2019. doi: 10.23939/istcmtm2019.04.00

[3] A. Arshad, R. Tasnim, A. H. M. Zahirul Alam, Sheros Khan, "Low value capacitance measurement system for the application of monitoring human body", ARPN Journal of Engineering and Applied Science, no. 11 (1), p. 327-330, 2016.

[4] D .Trushakov, S. Rendzinyak, I. Vasylchyshyn, "Determining of complex magnetic permeability of the ferromagnetic material by complex impedance of inductance coil with ferromagnetic core", Przeglad Elektrotechniczny, no. 4, p. 221-223, 2014. doi:10.12915/pe.2014.04.53

[5] J-E. Sigdell, "A principle for capacitance measurement, suitable for linear evaluation of capacitance transducers", IEEE Transaction on Instrumentation and Measurement, no. 1, p. 21-223, 1972.

[6] B. Bezprozvannych, I. Mirchuk, "The evaluation of possibility of normal operation of cables based on twisted pairs with PVC jacket under the conditions of high humidity and temperature", Electrical Engineering \& Electromechanics, no. 5, p. 49-53, 2017. doi: 10.20998/2074-272X.2017.5.08

[7] B. Bezprozvannych, A. Roginskiy, "Dielectric spectroscopy of casing thermosetting composite electrical insulation system of induction traction electric machines",
Electrical Engineering \& Electromechanics, no.1, p. 49-53, 2018. doi: 10.20998/2074-272X.2018.5.02

[8] M. Gutten, D. Korenciak, M. Sebok, et. al. "Diagnostics of transformer with insulation oil-paper", Przeglad Elektrotechniczny, no. 4, p. 69-72, 2015. doi:10.15199/48.2015.08.18

[9] L. Callegaro, "Electrical impedance: principles, measurement, and applications", Publishing House "CRC Press”, Boca Raton, USA, p. 92-138, 2013.

[10] M. Raven, D. Raven, "New approaches to the direct measurement of capacitance", Electrocomponent Science and Technology, no. 4, p. 37-42, 1977.

[11] A. Cichy, "Methods of synthesis of quasi-balanced circuits for measuring of impedance components", Elektronika ir Elektrotechnika, no.22 (2), p. 38-42, 2016. doi:10.2478/v10178-010-0022-8

[12] G. Lentka, "Using a particular sampling method for impedance measurement”, Metrol. Meas. Syst., no. 21 (3), p. 497-508, 2014. doi:10.2478/mms-2014-0042

[13] K. Chabowski, T. Piasecki, A. Dzierka, et. al., "Simple wide frequency range impedance meter based on AD5933 integrated circuit", Metrol. Meas. Syst., no. 22 (1), p. 13-24, 2015. doi:10.1515/mms-2015-0006

[14] P. Ramos, F. Janiero, A. Cruz Serra, et. al., "Recent developments on impedance measurements with DSPbased ellipse-fitting algorithms", IEEE Transactions on Instrumentation and Measurement, no. 58 (5), p. 1680-1689, 2009. doi:10.1109/TIM.2009.2014512

[15] P. Ramos, F. Janiero, "Implementation of DSP based algorithms for impedance measurement", in Proc. IEEE International Conference on Signal Processing and Communications, United Arab Emirates, 2007. doi:10.1109/ICSPC.2007.4728444

[16] P. Ramos, F. Janiero, T. Radil, "DSPIC-based impedance measuring instrument", Metrol. Meas. Syst., no. 18 (2), p. 185-198, 2011. doi:10.2478/v10178-011-0002-0

[17] P. Ramos, F. Janiero, T. Radil, "Comparative analysis of three algorithms for two-channel common frequency sinewave parameter estimation: ellipse fit, seven parameters sine fit and spectral sinc fit", Metrol. Meas. Syst., no. 17 (2), p. 250-270, 2010. doi:10.2478/v10178-010-0022-8

[18] R. Halir, J. Flusser, "Numerically stable direct least squares fitting of ellipses", in Proc. Conference in central Europe on computer graphics, visualization and interactive digital media, Czech Republic, 1998.

[19] E. Ventcel, Probability theory. State publishing house of physical and mathematical literature, Moscow, USSR, 1958.

[20] G. Bushina, Second order curves. Publishing House of Khabarovsk state technical univerciry, Khabarovsk, Russia, 1995.

[21] A. Kirkinskij, Linear Algebra and Analytical Geometry. Academic project, Moscow, Russia, 2006. 